\title{
Financial Disclosure
}

For: Paul W. Armstrong, Distinguished University Professor, University of Alberta, Founding Director, Canadian VIGOUR Centre, University of Alberta, Edmonton, Alberta ** Updated: September, 2018

\section{Monetary amounts are on an annual basis}

\begin{tabular}{|c|c|c|c|c|c|c|c|c|c|c|c|}
\hline \multirow[t]{2}{*}{ Company/(Companies): } & \multirow[t]{2}{*}{$\begin{array}{l}\text { Period } \\
\text { of } \\
\text { Support }\end{array}$} & \multirow[t]{2}{*}{ Name of Project } & \multirow{2}{*}{$\begin{array}{l}\text { 1) A research } \\
\text { grant or contract } \\
\text { from this } \\
\text { Company } \\
\text { generates } \\
\text { revenue for the } \\
\text { University of } \\
\text { Alberta }\end{array}$} & \multirow{2}{*}{$\begin{array}{l}\text { 2) A research } \\
\text { grant or } \\
\text { contract from } \\
\text { this Company } \\
\text { partially } \\
\text { supports my } \\
\text { research } \\
\text { projects }\end{array}$} & \multicolumn{2}{|c|}{$\begin{array}{l}\text { 3) Educational } \\
\text { activities or lectures } \\
\text { for this Company } \\
\text { generates revenue } \\
\text { for the University of } \\
\text { Alberta }\end{array}$} & \multicolumn{2}{|c|}{$\begin{array}{l}\text { 4) Consulting or } \\
\text { other services for } \\
\text { this company } \\
\text { generates personal } \\
\text { income }\end{array}$} & \multirow[t]{2}{*}{$\begin{array}{l}\text { 5) I receive } \\
\text { significant } \\
\text { personal } \\
\text { royalties from } \\
\text { this Company }\end{array}$} & \multirow[t]{2}{*}{$\begin{array}{l}\text { 6) I have } \\
\text { equity in } \\
\text { this } \\
\text { Company }\end{array}$} & \multirow[t]{2}{*}{ Comments } \\
\hline & & & & & $<\$ 10 \mathrm{~K}$ & $>\$ 10 \mathrm{~K}$ & $<\$ 10 \mathrm{~K}$ & $>\$ 10 \mathrm{~K}$ & & & \\
\hline $\begin{array}{l}\text { Sanofi-aventis Recherche \& } \\
\text { Développement }\end{array}$ & $\begin{array}{l}2012- \\
2018\end{array}$ & $\begin{array}{l}\text { A Randomized, Double-Blind, Placebo- } \\
\text { Controlled, Parallel-Group Study to } \\
\text { Evaluate the Effect of } \\
\text { SAR236553/REGN727 on the Occurrence } \\
\text { of Cardiovascular Events in Patients Who } \\
\text { Have Recently Experienced an Acute } \\
\text { Coronary Syndrome (ODYSSEY } \\
\text { Outcomes) }\end{array}$ & 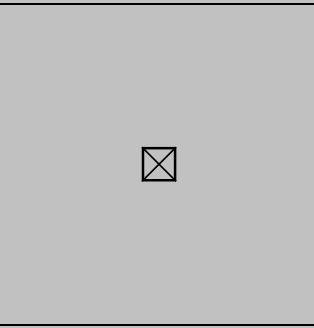 & $\square$ & $\square$ & $\square$ & $\square$ & $\square$ & $\square$ & $\square$ & \\
\hline AstraZeneca & $\begin{array}{l}2015- \\
2017\end{array}$ & $\begin{array}{l}\text { New Concepts in Acute Coronary } \\
\text { Syndromes: Beyond } 2000\end{array}$ & $\square$ & $\square$ & $\square$ & $\square$ & $\triangle$ & $\square$ & $\square$ & $\square$ & \\
\hline Merck & $\begin{array}{l}2015- \\
2017\end{array}$ & $\begin{array}{l}\text { New Concepts in Acute Coronary } \\
\text { Syndromes: Beyond } 2000\end{array}$ & $\square$ & $\square$ & $\square$ & $\square$ & $\bowtie$ & $\square$ & $\square$ & $\square$ & \\
\hline Merck & $\begin{array}{l}2016 \text { to } \\
\text { present }\end{array}$ & $\begin{array}{l}\text { VerICiguaT glObal study in patients with } \\
\text { heart failure and Reduced ejectIon } \\
\text { frAction (VICTORIA) }\end{array}$ & 区 & 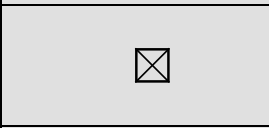 & $\square$ & $\square$ & $\square$ & 凶 & $\square$ & $\square$ & \\
\hline Bayer & $\begin{array}{l}2016- \\
2017\end{array}$ & $\begin{array}{l}\text { New Concepts in Heart Failure and Atrial } \\
\text { Fibrillation: Beyond } 2000\end{array}$ & $\square$ & $\square$ & $\square$ & $\square$ & 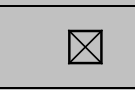 & $\square$ & $\square$ & $\square$ & \\
\hline Novartis & $\begin{array}{l}2016- \\
2017\end{array}$ & $\begin{array}{l}\text { New Concepts in Heart Failure and Atrial } \\
\text { Fibrillation: Beyond } 2000\end{array}$ & $\square$ & $\square$ & $\square$ & $\square$ & 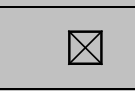 & $\square$ & $\square$ & $\square$ & \\
\hline Bayer & $\begin{array}{l}2017- \\
2019\end{array}$ & Consultant & $\square$ & $\square$ & $\square$ & $\square$ & $\square$ & $\nabla$ & $\square$ & $\square$ & \\
\hline Boehringer Ingelheim & $\begin{array}{l}2017 \text { to } \\
\text { present }\end{array}$ & $\begin{array}{l}\text { STrategic Reperfusion in elderly patients } \\
\text { Early After Myocardial Infaction } \\
\text { (STREAM-II) }\end{array}$ & $凶$ & $\square$ & $\square$ & $\square$ & $\square$ & $\square$ & $\square$ & $\square$ & \\
\hline
\end{tabular}




\begin{tabular}{|c|c|c|c|c|c|c|c|c|c|c|c|}
\hline \multirow[t]{2}{*}{ Company/(Companies): } & \multirow[t]{2}{*}{$\begin{array}{l}\text { Period } \\
\text { of } \\
\text { Support }\end{array}$} & \multirow[t]{2}{*}{ Name of Project } & \multirow{2}{*}{$\begin{array}{l}\text { 1) A research } \\
\text { grant or contract } \\
\text { from this } \\
\text { Company } \\
\text { generates } \\
\text { revenue for the } \\
\text { University of } \\
\text { Alberta }\end{array}$} & \multirow{2}{*}{$\begin{array}{l}\text { 2) A research } \\
\text { grant or } \\
\text { contract from } \\
\text { this Company } \\
\text { partially } \\
\text { supports my } \\
\text { research } \\
\text { projects }\end{array}$} & \multicolumn{2}{|c|}{$\begin{array}{l}\text { 3) Educational } \\
\text { activities or lectures } \\
\text { for this Company } \\
\text { generates revenue } \\
\text { for the University of } \\
\text { Alberta }\end{array}$} & \multicolumn{2}{|c|}{$\begin{array}{l}\text { 4) Consulting or } \\
\text { other services for } \\
\text { this company } \\
\text { generates personal } \\
\text { income }\end{array}$} & \multirow[t]{2}{*}{$\begin{array}{l}\text { 5) I receive } \\
\text { significant } \\
\text { personal } \\
\text { royalties from } \\
\text { this Company }\end{array}$} & \multirow[t]{2}{*}{$\begin{array}{l}\text { 6) I have } \\
\text { equity in } \\
\text { this } \\
\text { Company }\end{array}$} & \multirow[t]{2}{*}{ Comments } \\
\hline & & & & & $<\$ 10 K$ & $>\$ 10 K$ & $<\$ 10 \mathrm{~K}$ & $>\$ 10 \mathrm{~K}$ & & & \\
\hline Bayer & $\begin{array}{l}2018- \\
2020\end{array}$ & $\begin{array}{l}\text { A Randomized Parellel-Group, Placebo } \\
\text { Controlled, Double-Blind, Multi-Center } \\
\text { Confirmatory Trial to Evaluate the } \\
\text { Efficacy and Safety of the Oral sGC } \\
\text { Stimulator on Physical Limitations in } \\
\text { Patients with Heart Failure and Preserved } \\
\text { Ejection Fraction (VITALITY) }\end{array}$ & 凶 & $\square$ & $\square$ & $\square$ & $\square$ & 区 & $\square$ & $\square$ & \\
\hline CSL Limited & $\begin{array}{l}2018- \\
2022\end{array}$ & $\begin{array}{l}\text { A Phase 3, Multicenter, Double-blind, } \\
\text { Randomized, Placebo-controlled, Parallel- } \\
\text { group Study to Investigate the Efficacy } \\
\text { and Safety of CSL112 in Subjects with } \\
\text { Acute Coronary Syndrome (AEGIS II) }\end{array}$ & 区 & $\square$ & $\square$ & $\square$ & $\square$ & $\square$ & $\square$ & $\square$ & \\
\hline
\end{tabular}

** Please note this document should be placed in the context that Dr. Armstrong is working 0.5 FTE at the University. In accordance with the American Heart Association conflict of interest policies. 